\title{
Temperature Prediction Model of Cement Rotary Kiln Based on MPGA-LSSVM
}

\author{
Hui Zhao ${ }^{1,2, a}$, Yu Wang ${ }^{1, b}$, Hongjun Wang ${ }^{1, a}$, Youjun Yue \\ ${ }^{1}$ Tianjin Key Laboratory of Control Theory\& Applications in Complicated System, Tianjin University \\ of Technology, Tianjin 300384, China; \\ ${ }^{2}$ Tianjin Agricultural University, Tianjin 300384, China. \\ Correspondence author: Yu Wang, 15900237215@163.com. \\ ahongewang@126.com, ${ }^{\mathrm{b}} 15900237215 @ 163 . c o m,{ }^{\mathrm{c}}$ bakeryueyj@163.com
}

Keywords: Cement rotary kiln; Temperature prediction; Least square support vector machine; Multiple population genetic algorithm; MPGA-LSSVM.

\begin{abstract}
The burning zone temperature (BZT) in cement rotary kiln is a mostly important index which affects the quality of produced cement. In rotary kiln, the bilateral movements of air and complex chemical reactions take place. Thus, traditional modeling methods can not predict the BZT effectively. In order to improve the prediction accuracy, the temperature prediction model was established by least square support vector machine (LSSVM). The input variables of this model were selected by the gray correlation analysis method and mechanism analysis. The parameters of the LSSVM were optimized by multi population genetic algorithm (MPGA). The final simulation results show that the prediction accuracy is improved obviously. The effectiveness of the proposed method is verified.
\end{abstract}

\section{Introduction}

In the last fifty years, the world cement production has grown in a constant manner, both in the developed and developing countries. Producing cement requires large quantities of limestone and other material, like clay. With very high temperature, these mixed materials convert into cement. In fact, human operator observing the burning status is the widely applied method. But it is hard to directly measure the BZT due to complex chemical reactions. The reason has motivated the present work that, using intelligent algorithm, aims at acquiring an accurate model to predict the BZT. In [1], the author established a mathematical model through the conservation of mass and energy conservation theory. The artificial neural network ${ }^{[2]}$, fuzzy algorithm ${ }^{[3]}$ and support vector machine algorithm ${ }^{[4]}$ has been widely used in statistical modeling. The defects of these models are that prediction accuracy is not good enough and prediction time is too long. While least squares support vector machine (LSSVM) overcomes these disadvantages ${ }^{[5,6]}$. In this paper, least squares support vector machine is used to establish the temperature prediction model of cement rotary kiln, with multi population genetic algorithm (MPGA) optimizing its parameters. The simulation results show that the method is reasonable.

The paper is organized as follows. In the next Section, a description of cement production will be presented, which briefly describes the process. Then in Section 3 variables selection, modeling methods and modeling process design are introduced. In Section 4 the simulation results of MPGA-LSSVM will be presented. The paper is concluded in Section 5.

\section{Process Description}

Rotary kiln is the key equipment in cement industry used to convert calcareous raw material to cement clinkers. According to the temperature, the cement rotary kiln can be approximately divided into four parts: decomposition zone, reaction exothermic zone, burning zone and cooling zone. The decomposed raw material in decomposing furnace is fed into the rotary kiln from the kiln rear. Then the material goes along with the rotation of the rotary kiln, and it is gradually heated to $1450^{\circ} \mathrm{C}$. At 
this temperature, dicalcium silicate $(\mathrm{C} 2 \mathrm{~S})$ absorbs calcium oxide $(\mathrm{CaO})$ to form tricalcium silicate (C3S) that is the main component of cement clinker. During the burning process, the control of the temperature inside the kiln is crucial: insufficiently high temperatures in the kiln result in incompletely reacted products and poor-quality cement, while excessive maximum temperatures waste energy and damage kiln lining.

\section{PREDICTION MODEL OF BZT}

\subsection{Selection of Input Variables}

In this paper, qualitative analysis and quantitative analysis are conducted to select the variables.

1) Qualitative analysis

In cement rotary kiln system, in order to maintain the optimal heat distribution and achieve sustained, balanced and stable operation, the process parameters are required close attention. The main concerned parameters are feed rate of raw material (Mat), fuel feed rate (Fuel), current of motor $(\mathrm{Ka})$, rotational kiln speed $(\mathrm{Ks})$, back-end air pressure $(\mathrm{Bp})$, head-end air pressure $(\mathrm{Hp})$, back-end temperature (Bt), head-end temperature (Ht) and I.D fan speed (Fan).

Increasing Mat will increase the reactant in the rotary kiln and cause the BZT to decrease. Increasing Fuel will enhance the reaction in the rotary kiln, causing the BZT to rise. Ht indicates cooling air temperature and cooling air provides heat and oxygen for chemical reactions. With the rotating of rotary kiln, material moves from back-end to head-end. Increasing Ks will shorten the time. At the same time, material consumes less energy. Increasing Fan will provide sufficient air for combustion and speed up the reaction in the kiln. Bp and $\mathrm{Hp}$ indicate the ventilation in the kiln, and increasing BZT will increase $\mathrm{Bt}$ and $\mathrm{Ka}$.

2) Quantitative analysis

During the development of the systems, if the change trend is accordant, namely, the degree of changing synchronously is high, that the relational degree between the two factors is high, whereas it is low. So the Grey Relational Analysis method is a quantitative analysis method ${ }^{[7]}$. The principle is as follows:

Let $X$ be sequence set of variables' value, $x_{0} \in X$ be reference sequence of output value, $x_{i} \in X \quad$ be comparison sequence of input value.

$$
\begin{aligned}
& x_{0}(j)=\left\{x_{01}, x_{02}, \ldots, x_{0 n}\right\} \\
& x_{i}(j)=\left\{x_{i 1}, x_{i 2}, \ldots, x_{i n}\right\}
\end{aligned}
$$

where $i=1,2, \ldots, m, j=1,2, \ldots, n$. All of the reference sequences and comparison sequences are mean normalization. Firstly, the average value of each sequence is calculated. Secondly, the sequence is divided by average value of the sequence.

According to the formula (3), the correlative degree for each element is calculated.

$$
\xi_{0 i}(j)=\frac{\Delta_{\min }+\rho \Delta_{\max }}{\Delta_{0 i}(j)+\rho \Delta_{\max }}
$$

Where $\Delta_{0 i}(j)=\left|x_{0}(j)-x_{i}(j)\right|, \quad \Delta_{\max }=\min _{i} \min _{j}\left|x_{0}(j)-x_{i}(j)\right|, \quad \Delta_{\min }=\max _{i} \max _{j}\left|x_{0}(j)-x_{i}(j)\right|, \rho$ is differentiate coefficient, value arrangement $(0,1)$, generally $\rho=0.5^{[8]}$.

According to the formula (4), the correlative degree is calculated.

$$
r_{0 i}=\frac{1}{n} \sum_{j=1}^{n} \xi_{0 i}(j)
$$

The data of each variable is normalized, and the gray correlation value is calculated by the above formulas. The correlation coefficient is sorted from large to small. Usually, the variables, with correlation degree greater than 0.8 , are selected as the key variable. Correlation degree is shown in Table 1: 
Table 1 Correlation degree

\begin{tabular}{cc|cc}
\hline Variable name & Correlation degree & Variable name & Correlation degree \\
\hline Mat & 0.9605 & $\mathrm{Hp}$ & 0.7652 \\
Fuel & 0.9856 & $\mathrm{Ht}$ & 0.9657 \\
Ka & 0.9375 & $\mathrm{Bt}$ & 0.9840 \\
Ks & 0.9862 & Fan & 0.9883 \\
Bp & 0.6869 & - & - \\
\hline
\end{tabular}

All variables are selected except Bp and Hp.

\subsection{LSSVM Based on MPGA}

Least squares support vector (LSSVM) is improved for support vector machine (SVM). Compared with SVM, it reduces the complexity of calculation and inherits the advantages of SVM such as good generalization performance, globally optimal solution.

Given a training set of $N$ points $\left\{x_{k} ; y_{k}\right\}_{k=1}^{N}$, with the input values $x_{k} \in R^{m}$ and the output values $y_{k} \in R$. The optimization problem in original space is formulated as ${ }^{[9]}$ :

$$
\left\{\begin{array}{l}
\min J(\omega, e)=1 / 2 \omega^{T} \omega+1 / 2 \gamma \sum_{k=1}^{N} e_{k}^{2} \\
\text { s.t. } \quad y_{k}=\omega^{T} \varphi\left(x_{k}\right)+b+e_{k}
\end{array}\right.
$$

Where $\varphi(\cdot)$ is the nonlinear function which maps the input space into a higher dimensional feature space, $w$ is the weight vector, $e_{k}$ is error, $b$ denotes the bias and $\gamma$ is the regularization parameter which balances the complexity of the LSSVM model.

By introducing the Lagrange function to solve the optimal problem:

$$
L(w, b, e, \alpha)=J(w, e)-\sum_{k=1}^{N} \alpha_{k}\left\{w^{T} \varphi\left(x_{k}\right)+b+e_{k}-y_{k}\right\}
$$

where $\alpha_{k} \in R(k=1,2, \ldots, N)$ are Lagrange multipliers.

Constructed kernel function: $K\left(x_{k}, x_{l}\right)=\varphi\left(x_{k}\right)^{T} \varphi\left(x_{l}\right)$. In this paper, we select Radial Basis Function (RBF) kernel function ${ }^{[10]}: K\left(x, x_{k}\right)=\exp \left(-\left\|x-x_{k}\right\|^{2} / 2 \sigma^{2}\right)$.

Finally, the regression function is expressed as:

$$
y(x)=\sum_{k=1}^{N} \alpha_{k} K\left(x, x_{k}\right)+b
$$

There are two parameters in LSSVM. If chosen by experience, we can't get the best result. Multi population genetic algorithm (MPGA) is the powerful stochastic algorithm based on the principles of natural selection and natural genetics ${ }^{[11,12]}$. In the search process, MPGA has better global search capability. Using the algorithm optimize the parameters of least squares support vector machine $\gamma$ and $\sigma$. The specific steps that selecting the parameters by MPGA are as follows:

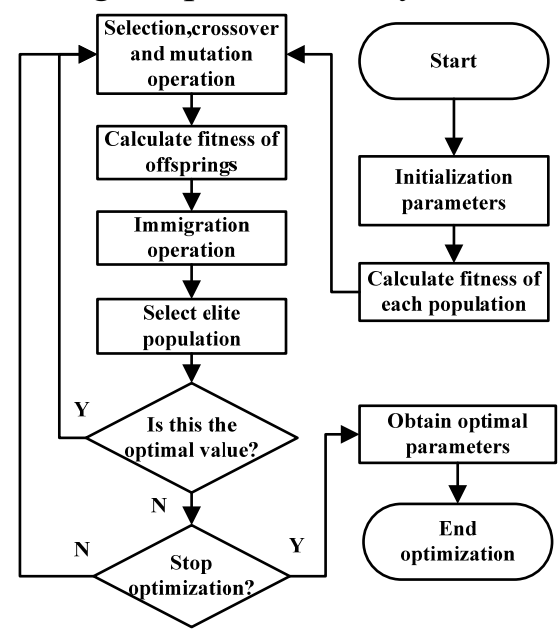

Fig.1 Parameter selection

\subsection{Temperature Model of Cement Rotary Kiln}

The least squares support vector machine modeling based on a variety of genetic algorithm 
optimization is as follows:

1) With the analysis of the factors relating to BZT and calculating the correlation degree, seven process parameters are selected: feed rate of raw material, fuel feed rate, current of motor, rotational kiln speed, back-end temperature, head-end temperature and I.D fan speed, which are represented by $x_{1}-x_{7} .100$ groups of data are randomly selected from the database. 80 groups of data are randomly selected as training set and others are used as test set. Using following formula to normalize data:

$$
\hat{x}_{i j}=\frac{x_{i j}-x_{i \min }}{x_{i \max }-x_{i \min }}
$$

2) Multi population genetic algorithm initialization. Population quantity is 4 , and the number of individuals in each population is 30 . Crossover and mutation probability are randomly obtained at $(0.7,0.9)$ and $(0.001,0.05)$. The fitness function is the mean square error between the test set and the training set.

3) Using multi population genetic algorithm find the best value of $\gamma$ and $\sigma$. The temperature model of cement rotary kiln is established by the training set data and the generalization ability of the model is tested by the test set data.

\section{Simulation and Analysis}

In this paper, the production data of cement plants are used as samples. The optimal interval of $\gamma$ is respectively $(0.01,10000)$ and $\sigma$ is $(0.1,1000)$. The optimization results are 10000 and 18.555 . The fitness curve of each group is shown in Fig. 2.

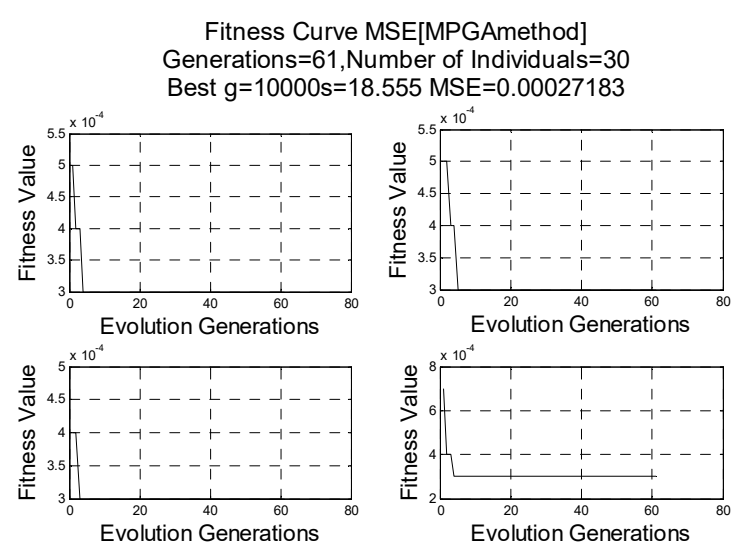

Fig. 2 Fitness curve of each population

The fitness curve of the four populations converges after five or six generations. It indicates that the searching speed is fast. The reason for this result is that there is immigration between the populations. After independent optimizing, the best individual of previous population replaces the worst one of next population. In this way, link between populations is strengthening, and the poor individuals are eliminated. Therefore, it is easier to find the optimal value by multi population genetic algorithm.

The temperature model is established by optimized LSSVM, common LSSVM and BP neural network. The simulation results of the three soft sensors are shown in Fig. 4 and Fig. 5, where 80 samples correspond to training data and 20 samples correspond to testing data. 


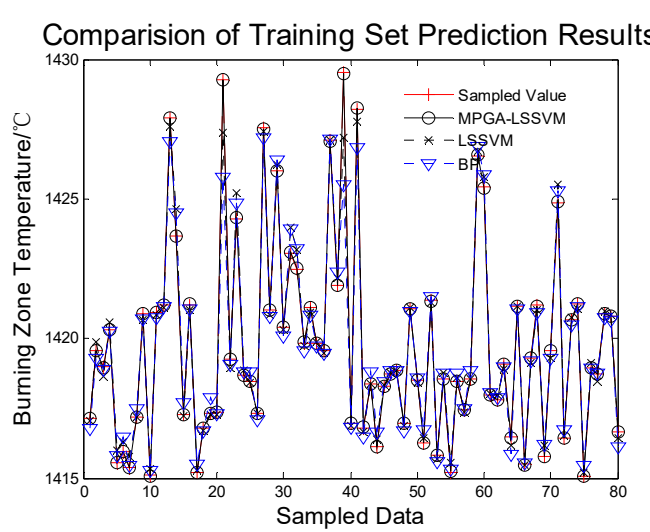

Fig. 4 Training set prediction results

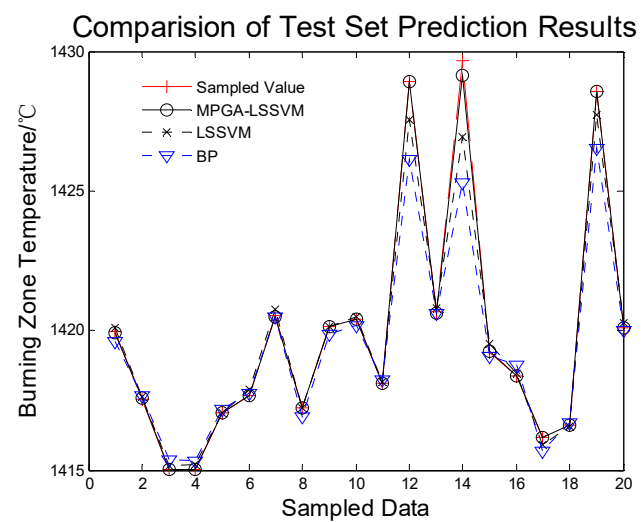

Fig. 5 Test set prediction results

To measure the performances of the three soft sensors, the targets of coefficient of determination $\left(\mathrm{R}^{2}\right)$, mean square error (MSE) and mean absolute error (MAE), mean relative error (MRE) are calculated. The calculation results and computing time of the three soft sensors are listed in Table 2.

$$
\begin{aligned}
& R^{2}=\frac{\left(l \sum_{i=1}^{l} \hat{y}_{i} y_{i}-\sum_{i=1}^{l} \hat{y}_{i} \sum_{i=1}^{l} y_{i}\right)^{2}}{\left(l \sum_{i=1}^{l} \hat{y}_{i}^{2}-\left(\sum_{i=1}^{l} \hat{y}_{i}\right)^{2}\right)\left(l \sum_{i=1}^{l} y_{i}^{2}-\left(\sum_{i=1}^{l} y_{i}\right)^{2}\right)} \\
& M S E=\frac{1}{l} \sum_{i=1}^{l}\left(\hat{y}_{i}-y_{i}\right)^{2} \\
& M A E=\frac{1}{l} \sum_{i=1}^{l}\left|\hat{y}_{i}-y_{i}\right| \\
& M R E=\frac{1}{l} \sum_{i=1}^{l} \frac{\left|\hat{y}_{i}-y_{i}\right|}{y_{i}}
\end{aligned}
$$

Table 2 The prediction errors of each model

\begin{tabular}{ccccc}
\hline & MSE & R2 & MAE & MRE \\
\hline MPGA-LSSVM & $2.6585 \times 10^{-4}$ & 0.9994 & 0.0397 & $2.7841 \times 10^{-5}$ \\
LS-SVM & $9.9 \times 10^{-3}$ & 0.9872 & 0.3503 & $2.4565 \times 10^{-4}$ \\
BP & $3.06 \times 10^{-2}$ & 0.9735 & 0.6426 & $4.5067 \times 10^{-4}$ \\
\hline
\end{tabular}

Where $\hat{y}_{i}$ are samples of real value, $y_{i}$ are samples of predicted value and $i=1,2, \ldots, l$.

From Table 2, we can see that errors of the proposed MPGA-LSSVM model are lower than the other two models. It can be concluded that the MPGA-LSSVM model shows outstanding performances than common LSSVM model and BPNN model with better learning and regeneration capabilities.

\section{Conclusion}

This paper presents a method to optimize parameters of least square support vector machine based on multi population genetic algorithm. Immigration operator increases information exchange among populations, and elimination of the worst individuals ensure the population quality. The artificial selection of elite population preserves the best individual, so that the optimal value can be obtained quickly. The comparison of simulation results and targets among three models indicates that MPGA-LSSVM has higher accuracy.

\section{Acknowledgment}

This research is supported by the Tianjin Municipal Science and technology support program (13ZCZDGX03800). 


\section{References}

[1] Zhao J. The research of modeling and control of cement rotary kiln system[D]. Zhengzhou: Zhengzhou University, 2014

[2] Baosheng Yang; Xiushui Ma; Qian Zhang. Elman Neural Network based Temperature Prediction in Cement Rotary Kiln Calcining Process[C]// Intelligent Systems and Knowledge Engineering (ISKE), 2010 International Conference on,596-600

[3] Cai H M, Zhang G L. Study on rotary kiln temperature control system based on fuzzy PID control algorithm[J]. Modern Electronics Technique, 2015,23:167-170.

[4] Jiang H Y, Wang X D, Zhou X J, Chai T Y. Study on Soft Sensor for Temperature of Burning Zone Based on SVR[J]. Journal of System Simulation, 2008,11:2951-2955.

[5] Mohammad Ali Ahmadi, Behnam Mahmoudi. Development of robust model to estimate gas oil interfacial tension using least square support vector machine: Experimental andmodeling study[C]// The Journal of Supercritical Fluids, Volume 107, January 2016, Pages 122-128

[6] Chang A Y, Wu T J, Zhao H, etc. Prediction and Analysis of Pulverized Coal Ignition Temperature Based on a Least Square Supportive Vector Machine[J]. Journal of Engineering for Thermal Energy and Power. 2011,01:97-99+126-127.

[7] Tian M, Liu S F, Bo Z K. Research on grey relational degree algorithm model[J]. Statistics \& Decision, 2008,01:24-27.

[8] C. Jie; D. Yao-guo. Study on affine property of some algorithms for grey relational analysis[C]// 2009 IEEE International Conference on Grey Systems and Intelligent Services (GSIS 2009) $52-56$

[9] Yan G. Research of Aluminum Electrolysis Process Modeling and Control Based on Least Squares Support Vector Machine[D]. Changsha : Central South University, 2012.

[10]Lv H, Zhou C, Zou J, etc. Genetic algorithm based on multi-population evolution[J]. Computer Engineering and Applications. Computer Engineering and Applications, 2010, 46 (28) : 57-60.

[11]Li M Q. Basic Theory and Application of Genetic Algorithm[M]. Beijing : Science Press, 2012

[12]Qin W N. A genetic algorithm research based on multi-population[D]. Jinan : Shandong Normal University, 2009. 